\title{
Resolving Anharmonic Lattice Dynamics in Molecular Crystals with X-Ray Diffraction and Terahertz Spectroscopy
}

\author{
Martin Hutereau $\odot,{ }^{1,2,3}$ Peter A. Banks $\odot,{ }^{4}$ Ben Slater, ${ }^{1}$ J. Axel Zeitler $\odot,{ }^{3}$ Andrew D. Bond $\odot,{ }^{2}$ and Michael T. Ruggiero $\odot^{3,4, *}$ \\ ${ }^{1}$ Department of Chemistry, University College London, 20 Gordon Street, London WC1H OAJ, United Kingdom \\ ${ }^{2}$ Department of Chemistry, University of Cambridge, Lensfield Road, Cambridge CB2 1EW, United Kingdom \\ ${ }^{3}$ Department of Chemical Engineering and Biotechnology, University of Cambridge, \\ Philippa Fawcett Drive, Cambridge CB3 OAS, United Kingdom \\ ${ }^{4}$ Department of Chemistry, University of Vermont, 82 University Place, Burlington, Vermont 05405, USA
}

(Received 4 December 2019; revised 6 February 2020; accepted 31 July 2020; published 31 August 2020)

\begin{abstract}
Molecular crystals are increasingly being used for advanced applications, ranging from pharmaceutics to organic electronics, with their utility dictated by a combination of their three-dimensional structures and molecular dynamics - with anharmonicity in the low-frequency vibrations crucial to numerous bulk phenomena. Through the use of temperature-dependent $\mathrm{x}$-ray diffraction and terahertz time-domain spectroscopy, the structures and dynamics of a pair of isomeric molecular crystals exhibiting nearly free rotation of a $\mathrm{CF}_{3}$ functional group at ambient conditions are fully characterized. Using a recently developed solid-state anharmonic vibrational correction, and applying it to a molecular crystal for the first time, the temperature-dependent spatial displacements of atoms along particular terahertz modes are obtained, and are found to be in excellent agreement with the experimental observations, including the assignment of a previously unexplained absorption feature in the low-frequency spectrum of one of the solids.
\end{abstract}

DOI: 10.1103/PhysRevLett.125.103001

The interplay between molecular structure, threedimensional packing, and lattice dynamics is critical to the proper function and behavior of condensed phase materials [1-5]. In organic materials, these forces are responsible for countless properties, such as crystalline polymorphism, phase transformation phenomena, semiconducting ability, physical stability, and so on [6-9]. For over a century, x-ray crystallography has been the primary method of determining the structures of crystalline materials, while low-frequency vibrational spectroscopy $\left(0.3-3 \mathrm{THz}, 10-100 \mathrm{~cm}^{-1}\right)$ has emerged over the past few decades as a powerful technique for understanding the lattice dynamics of such solids [10]. Yet, while both of these techniques are the principal means of characterizing structure and dynamics, respectively, they are complements in that they both indirectly probe the processes of the other. For example, $\mathrm{x}$-ray crystallography provides the average spatial distribution of electron density, which inherently includes low-frequency vibrations that are highly excited at ambient conditions $\left(k_{B} T_{298} \approx 6.2 \mathrm{THz} \approx 207 \mathrm{~cm}^{-1}\right)$. On the other hand, low-frequency vibrational spectroscopy is directly tied to the structures of solids, as the large-amplitude vibrational motions are largely dictated by long-range forces from neighboring molecules and the three-dimensional periodic nature of the solid $[11,12]$. Additionally, low-frequency modes are notorious for being highly anharmonic, and thus there exists a distinct need to understand how anharmonicity manifests in solid-state structures and dynamics [13,14].

To date, there has been little work to connect static diffraction techniques with dynamic spectroscopic techniques, although recent efforts have highlighted the significantly detailed information that can be obtained by using these techniques in tandem. For example, Kozina et al. have used terahertz pump-X-ray probe measurements to characterize anharmonic coupling in inorganic crystals [15], opening the door to understanding vibrational relaxation through the analysis of purely structural information.

Understanding the origins of low-frequency vibrational motions can therefore have a profound effect on interpreting crystallographic data, with one specific example being significant thermal disorder of atoms or functional groups within crystals - a direct result of vibrational anharmonicity. While solid-state methyl rotors have been of interest for their ability to sense a wide variety of weak forces [16-19], they are difficult to interpret using $\mathrm{x}$ rays due to the lowscattering power of hydrogen atoms. On the other hand, trifluoromethyl $\left(\mathrm{CF}_{3}\right)$ groups should be more easily observed with $\mathrm{x}$ rays, and thereby provide a mechanism for understanding these effects with high spatial resolution.

Crystals of two isomers containing trifluoromethyl groups were used as the basis for this study: 2-nitro4-(trifluoromethyl)benzoic acid (2NTFB) and 4-nitro-2(trifluoromethyl)benzoic acid (4NTFB). The structures 


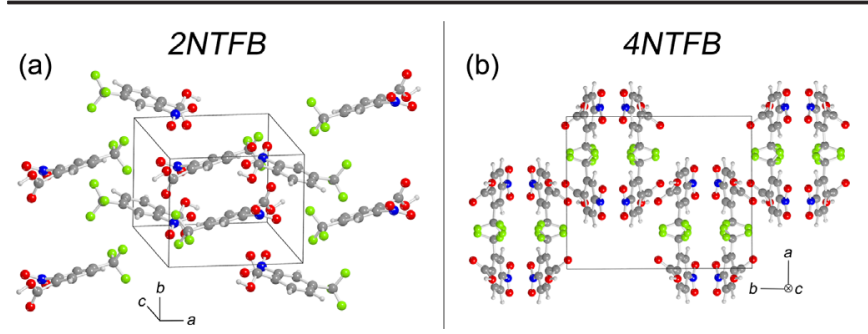

FIG. 1. Structures of the two NTFB crystals. (a) The unit cell for $2 \mathrm{NTFB}$, showing the interdigitated $\mathrm{CF}_{3}$ groups along the $b$ axis. (b) The unit cell for $4 \mathrm{NTFB}$, showcasing the $\mathrm{CF}_{3}$ ribbons along the [004] Miller plane. There are 8 f.u. per cell in 4NTFB, compared to 4 for 2 NTFB. The elements are colored accordingly: $\mathrm{C}$ is gray, $\mathrm{O}$ is red, $\mathrm{N}$ is blue, $\mathrm{F}$ is green, and $\mathrm{H}$ is white.

(Fig. 1) were determined for the first time for this study using single-crystal X-ray diffraction (XRD) over the temperature range 120-320 K. At ambient conditions (300K), the fluorine atoms of the $\mathrm{CF}_{3}$ group in 2NTFB show large displacement ellipsoids that suggest significant rotation about the $\mathrm{C}-\mathrm{C}$ bond. In contrast, the ellipsoids in 4NTFB are much smaller, indicative of more restrictive motion. In order to more precisely characterize the rotational motion of the $\mathrm{CF}_{3}$ group, electron density profiles through a plane formed by the three fluorine atoms were generated by characterizing the Fourier transform of the XRD structure factors (additional technical details provided in the Supplemental Material [20]). Figure 2 shows the XRD-determined electron density about the plane of the $\mathrm{CF}_{3}$ ring as a function of angle, and the evolution of the density distribution with temperature. As the temperature is lowered, flatter regions between the maxima (indicating that the $\mathrm{F}$ atoms are sufficiently localized around their static positions) first become clearly evident for $2 \mathrm{NTFB}$ around $140 \mathrm{~K}$. In $4 \mathrm{NTFB}$, the maxima are more clearly separated at all temperatures, and secondary peaks indicative of an alternative localized position for the $\mathrm{CF}_{3}$ group, rotated by $60^{\circ}$ relative to the main position, are evident at around $180 \mathrm{~K}$ and below. This likely indicates that a small number of molecules within the bulk access an

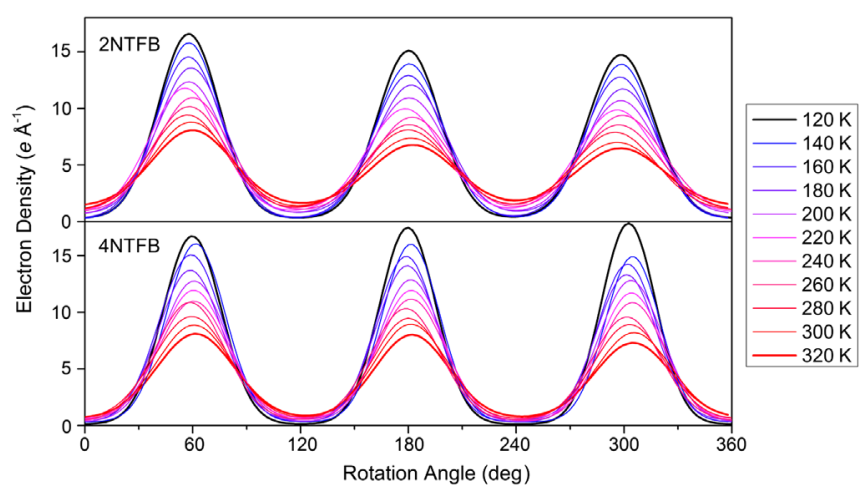

FIG. 2. Temperature overlay of the electron density distribution along the $\mathrm{CF}_{3}$ ring for 4NTFB and 2NTFB. energetically similar, yet distinct conformation. Clearly, these observations highlight a difference between the rotational potentials for the two molecules in the crystalline environment.

In an effort to decouple internal torsional motion from overall molecular translation and libration, the x-ray data were further analyzed using the translation-libration-screw (TLS) approach [29]. Details of the calculations are provided in the Supplemental Material [20]. The analysis was carried out first assuming entirely rigid molecules, then again treating the $\mathrm{CF}_{3}$ group as an attached rigid group able to rotate relative to the remainder of the molecule. For $4 \mathrm{NTFB}$, the overall fit to the atomic displacement parameters is very closely comparable using fully rigid molecules or allowing internal motion, consistent with limited internal torsional motion of the $\mathrm{CF}_{3}$ group. For $2 \mathrm{NTFB}$, the fit to the atomic displacement parameters improves significantly with the introduction of internal motion, again consistent with the other indications of greater torsional motion in this case. The TLS calculation provides the overall torsional libration around the $\mathrm{C}-\mathrm{CF}_{3}$ axis, which comprises both the component of the molecular libration as a whole and any relative motion of the $\mathrm{CF}_{3}$ group. This overall value is roughly in the range $11^{\circ}-20^{\circ}$ for $2 \mathrm{NTFB}$, compared to $0^{\circ}-3^{\circ}$ for $4 \mathrm{NTFB}$, increasing linearly over the temperature range in both cases (see Supplemental Material [20]).

The structures of the two crystals are shown in Fig. 1 and are provided in electronic (CIF) format in the Supplemental Material [20]. In both solids, the common intermolecular hydrogen bonded benzoic acid dimer motif is present. However, the packing of the two isomers results in distinctly different crystalline environments for the $\mathrm{CF}_{3}$ groups. In $2 \mathrm{NTFB}$, one $\mathrm{F}$ atom is involved in an approximately linear $\mathrm{C}-\mathrm{H} \cdots \mathrm{F}$ interaction, while the other two $\mathrm{F}$ atoms point toward the $\mathrm{COOH}$ groups of neighboring molecular dimers. In 4NTFB, two $F$ atoms accept $\mathrm{C}-\mathrm{H} \cdots \mathrm{F}$ interactions, while the third points between neighboring molecules, making no clear intermolecular contact. The shortest distances between $\mathrm{CF}_{3}$ groups in 2NTFB have $\mathrm{C} \cdots \mathrm{C}=4.322(2) \AA$ (at $120 \mathrm{~K}$ ), with the groups aligned to form a single offset strand along the crystallographic $b$ axis. In 4NTFB, the $\mathrm{C} \cdots \mathrm{C}$ distances are larger [4.474(6) and 5.092(2) $\AA$ at $120 \mathrm{~K}]$ and the $\mathrm{CF}_{3}$ groups adopt a double-strand arrangement along the $b$ axis. We note that these intermolecular contacts are much larger than the respective elements van der Waals radii of ca. 1.70 and $1.47 \AA$.

Analysis of the crystal structures provides little insight into the nature of the forces that are present in these materials. In order to probe these, experimental terahertz time-domain spectroscopy (THz TDS) experiments, which probe the low-frequency vibrational motions directly, were performed using a commercial Terapulse THz TDS system (TeraView Ltd., Cambridge, UK). Samples were prepared 

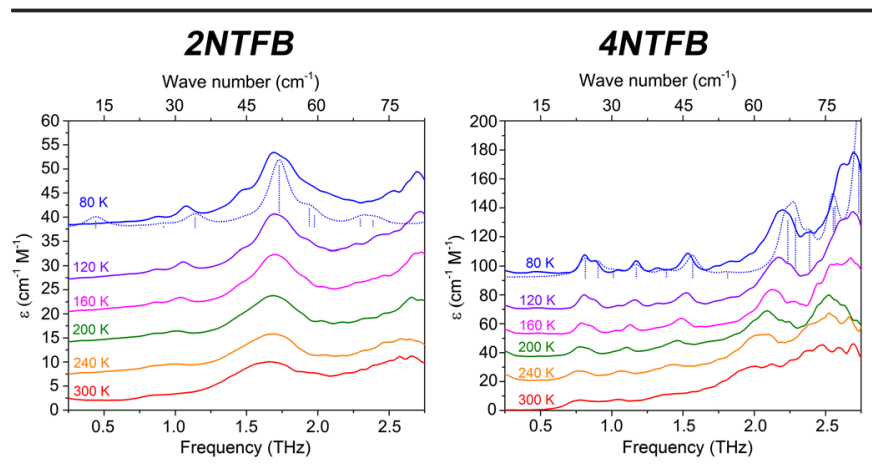

FIG. 3. Temperature overlay of the terahertz spectra for 2NTFB and 4NTFB. The curves have been offset from each other to aid visualization. The dotted curves represent the predicted spectra, which have been convolved with Lorentzian line shapes.

for $\mathrm{THz}$ TDS experiments by mixing the as-received commercially available material with high-density polyethylene to a $10 \% \mathrm{w} / \mathrm{w}$ concentration, followed by grinding and pressing into 13 -mm-diameter freestanding pellets with a thickness of $2 \mathrm{~mm}$ (additional technical details provided in the Supplemental Material [20]). The THz TDS spectra of both isomers are presented in Fig. 3, and they exhibit distinct spectral signatures that are strongly influenced by temperature.

Figure 3 shows at least four major absorption features for 2NTFB $(80 \mathrm{~K})$ at $0.91,1.09,1.49$, and $1.75 \mathrm{THz}$. In contrast, for the same temperature, 4NTFB exhibits at least 7 identifiable peaks, at $0.83,0.90,1.03,1.19,1.31,1.53$, and $2.20 \mathrm{THz}$. The greater number of features in 4NTFB is likely a result of the increased number of atoms in the unit cell, which results in a larger number of IR-active modes. The differences between these spectral signatures highlight how different the long-range forces in these two crystals are. The spectra are both strongly influenced by temperature, with all of the features sharpening and shifting to higher frequencies as the temperature is lowered. While this is common for molecular crystals [14,30,31], the features at 1.75 and $2.20 \mathrm{THz}$ in $2 \mathrm{NTFB}$ and 4NTFB, respectively, exhibit a much larger change in both the frequency and intensity compared to the other modes.

In order to assign the experimental spectra, vibrational simulations were performed using CRYSTAL17 [32] with the Grimme-D3-BJ dispersion corrected PBE [33-35] functional and Ahlrich's VTZP basis set [36]. Prior to performing the vibrational analysis, the structures of the two solids were fully optimized without constraints (i.e., lattice parameters and atomic positions), which resulted in an average absolute error in unit cell lengths and bond distances of $<0.5 \%$ for both solids (compared to the $120 \mathrm{~K}$ structures). The results of the vibrational simulations, shown in Fig. 3, represent an excellent agreement with the experimental results for both crystals, although it was necessary to scale the frequency of the simulated spectra for $2 \mathrm{NTFB}$ by a factor of 0.9 , likely due to this crystal exhibiting more pronounced anharmonicity [37,38]. A notable exception to the spectral agreement, however, is the predicted feature at $0.421 \mathrm{THz}$ in $2 \mathrm{NTFB}$, which does not appear to have a corresponding peak in the experimental spectrum, even when the concentration of the sample was greatly increased. It is interesting to note that this particular mode represents hindered out-of-phase rotation of the $\mathrm{CF}_{3}$ groups with a transition moment in the $a c$ crystallographic plane-the in-phase mode is IR inactive due to symmetry, but occurs at $0.41 \mathrm{THz}$, nearly the same frequency thus indicating only minor coupling of the $\mathrm{CF}_{3}$ groups (full mode assignment available in the Supplemental Material [20]). In order to investigate this discrepancy further, an explicit anharmonic calculation of the vibrational mode was performed by manually displacing the crystal along the normal mode coordinate and calculating the energy at each step. The potential generated, shown in Fig. 4, deviates significantly from the harmonic approximation prediction, with the mode being very shallow at small displacements but then rising rapidly (and thus deviating from quadratic behavior) with larger displacements. This is due to the solid-state nature of the sample, where large displacements result in interactions that are strongly repulsive due to the proximity of neighboring molecules. This new potential was used to determine the true anharmonic frequency using the recently implemented vibrational self-consistent field routine $[39,40]$, which shifted the mode by almost $1 \mathrm{THz}$ so that it now accounts for the vibrational feature occurring in the experimental spectrum at $1.49 \mathrm{THz}$. Interestingly, the mode corresponding to the $\mathrm{CF}_{3}$ rotation in $4 \mathrm{NTFB}$ is predicted to occur at $2.28 \mathrm{THz}$, which is in good agreement with the experimental spectrum, indicating that it exhibits a much more harmonic behavior.

With the aim of exploring the rotational motion more deeply, the dihedral angle corresponding to the $\mathrm{CF}_{3}$ rotation was manually scanned in $10^{\circ}$ increments and the energy calculated at each step for both materials. The generated potential for 2NTFB is very similar in shape and magnitude to the anharmonic vibrational potential of the terahertz mode at $1.49 \mathrm{THz}$. This potential enables estimation of the absolute value of the displacement of the fluorine atoms as a function of vibrational excitation, with the zero-point motion corresponding to an approximate $5^{\circ}$ displacement $\left(1.5 \mathrm{THz}=0.598 \mathrm{~kJ} \mathrm{~mol}^{-1}\right)$. The barrier is approximately $61.5 \mathrm{~kJ} \mathrm{~mol}^{-1}$ (corresponding to a temperature of $30000 \mathrm{~K}$ ) suggesting that the fluorine atoms rarely, if ever, exchange positions, and that the $\mathrm{CF}_{3}$ group in 2NTFB is truly a hindered rotor. In order to obtain the average temperature-dependent displacement of the $\mathrm{CF}_{3}$ group, it was assumed that the potential contained a large number of closely spaced energy levels, such that a classical approximation could be applied to find the probability density of the fluorine atom positions for a given temperature: 

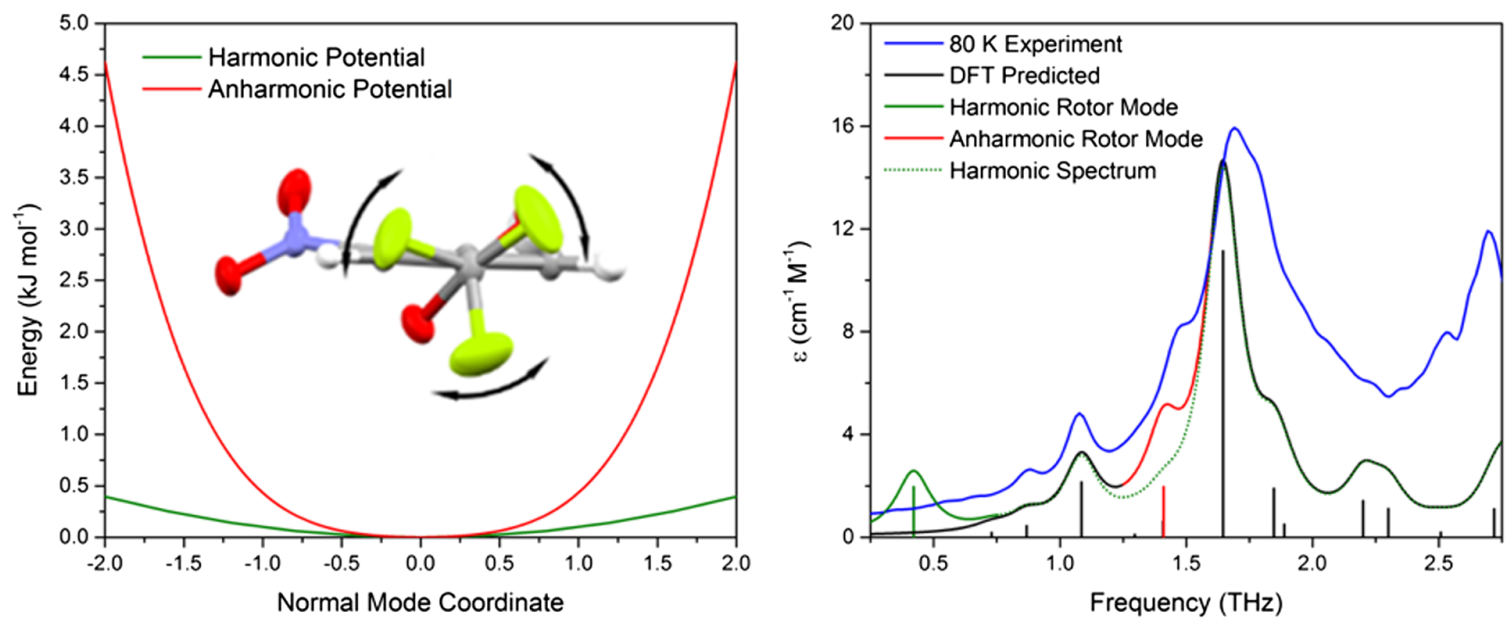

FIG. 4. Harmonic (green) and explicit anharmonic (red) simulations for the $\mathrm{CF}_{3}$ rotational mode in $2 \mathrm{NTFB}$. Left: the vibrational potentials for the harmonic and anharmonic case, with the arrows in the inset representing the normal mode coordinate. Right: the $80 \mathrm{~K}$ experimental THz TDS (blue), and the simulated vibrational spectra. The new position of the vibrational transition using the anharmonic potential (red peak), and the original harmonic feature (green) along with the entire harmonically determined spectrum (dotted green curve).

$$
P(\theta)=\frac{e^{-V(\theta) / k_{B} T}}{\int_{-60}^{60} e^{-V(\theta) / k_{B} T} d \theta}
$$

where $V(\theta)$ is the rotational potential, $k_{B}$ is Boltzmann's constant, and $T$ is the absolute temperature. The full width at half maximum (FWHM) of these distributions can be compared to those derived from the plots of the $\mathrm{CF}_{3}$ electron density at every temperature for which $\mathrm{x}$-ray results were obtained, see Fig. 2. Since the calculated probability density applies to the fluorine atom positions, it is necessary to add a correction (constant as a function of $T$ ) to yield the electron density distribution. This finite size was determined within this geometry to be approximately $23.7^{\circ}$, using the approximation of a solid fluorine atom with radius equal to the covalent radius for simplicity. It is important to note that fluorine is notorious for having a highly variable radius; however, the gradient of the two curves is nearly the same, indicating that the investigated phenomena (the change in distribution as a function of temperature) is consistent between the two techniques. The resulting widths are compared in Fig. 5, and although the absolute values differ, the gradients are very similar. These results are also mirrored in the $\mathrm{THz}$ TDS spectra, where the features corresponding to the $\mathrm{CF}_{3}$ motion exhibit a much more pronounced temperature dependence than the other modes. This is likely due to a large number of different chemical environments, which leads to slightly different vibrational transitions and thus a more diffuse mode with increasing "disorder" as temperature is increased.

Overall, this work highlights the deep connection between anharmonic low-frequency vibrational motions and corresponding crystallographic structures as a function of temperature, and the value that THz TDS spectroscopy can add to variable-temperature x-ray crystallographic measurements. The ability to relate a single low-frequency vibration directly to the observed large-magnitude displacement information in the x-ray structures indicates that
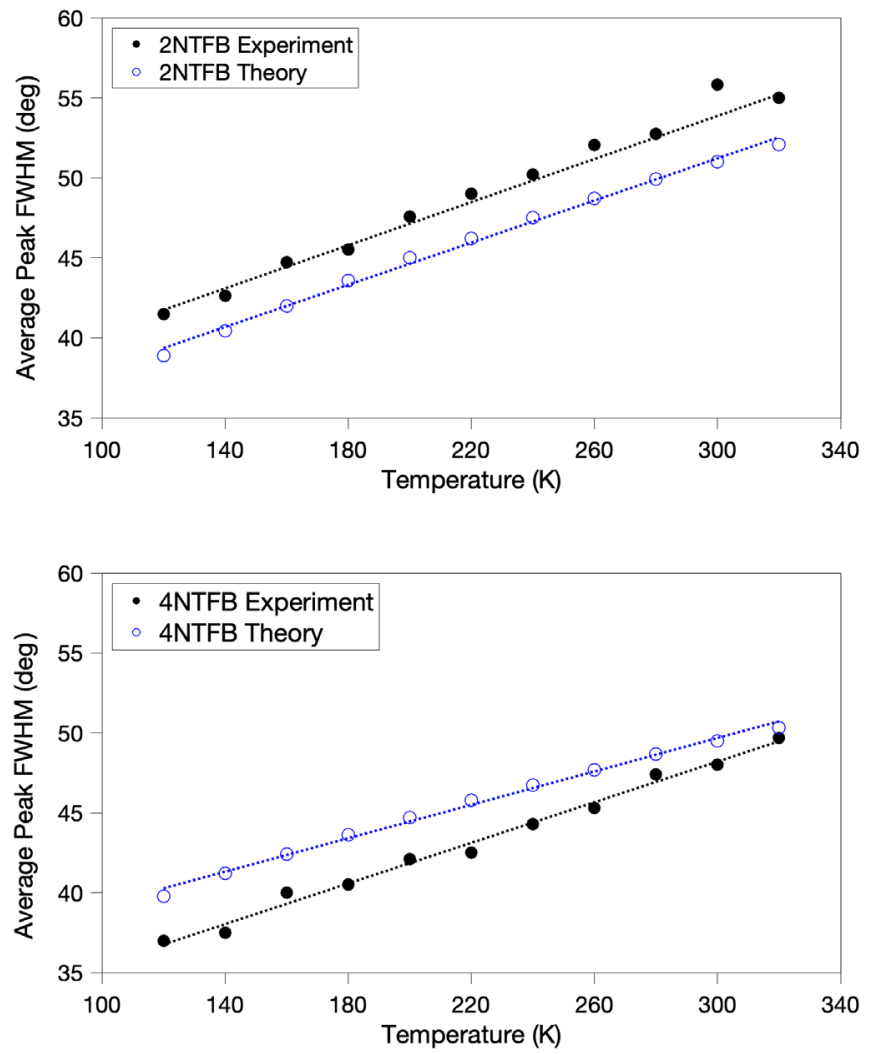

FIG. 5. Comparison between the electron density distribution FWHMs derived from x-ray experiments and those derived computationally for 2NTFB (top) and 4NTFB (bottom). 
the applied methods can describe such phenomena accurately, and thereby contribute significantly to our understanding of the dynamical aspects of crystals, specifically anharmonic low-frequency dynamics. Finally, the success of recently developed anharmonic corrections to vibrational potentials represents a step forward in characterizing similar phenomena in related materials.

M. T. R. and P. A. B. thank the University of Vermont for support. M.H., M.T.R., and J.A.Z. thank the UK Engineering and Physical Sciences Research Council for funding (EP/N022769/1).

*Corresponding author. michael.ruggiero@uvm.edu

[1] U. Scherf and E. J. W. List, Adv. Mater. 14, 477 (2002).

[2] A. Mishra, M. K. R. Fischer, and P. Bäuerle, Angew. Chem., Int. Ed. Engl. 48, 2474 (2009).

[3] E. Ostuni, R. G. Chapman, R. E. Holmlin, S. Takayama, and G. M. Whitesides, Langmuir 17, 5605 (2001).

[4] C. E. Wilmer, O. K. Farha, Y.-S. Bae, J. T. Hupp, and R. Q. Snurr, Energy Environ. Sci. 5, 9849 (2012).

[5] K. A. Niessen, M. Xu, A. Paciaroni, A. Orecchini, E. H. Snell, and A. G. Markelz, Biophys. J. 112, 933 (2017).

[6] K. Ostrowska, M. Kropidłowska, and A. Katrusiak, Cryst. Growth Des. 15, 1512 (2015).

[7] E. P. J. Parrott, N. Y. Tan, R. Hu, J. A. Zeitler, B. Z. Tang, and E. Pickwell-MacPherson, Mater. Horiz. 1, 251 (2014).

[8] M. T. Ruggiero, J. J. Sutton, S. J. Fraser-Miller, A. J. Zaczek, T. M. Korter, K. C. Gordon, and J.A. Zeitler, Cryst. Growth Des. 18, 6513 (2018).

[9] M. T. Ruggiero, W. Zhang, A. D. Bond, D. M. Mittleman, and J. A. Zeitler, Phys. Rev. Lett. 120, 196002 (2018).

[10] E. P. J. Parrott and J. A. Zeitler, Appl. Spectrosc. 69, 1 (2015).

[11] P. M. Hakey, D. G. Allis, W. Ouellette, and T. M. Korter, J. Phys. Chem. A 113, 5119 (2009).

[12] M. R. C. Williams, D. J. Aschaffenburg, B. K. Ofori-Okai, and C. A. Schmuttenmaer, J. Phys. Chem. B 117, 10444 (2013).

[13] M. D. King, W. D. Buchanan, and T. M. Korter, J. Phys. Chem. A 114, 9570 (2010).

[14] M. T. Ruggiero, J. A. Zeitler, and A. Erba, Chem. Commun. 53, 3781 (2017).

[15] M. Kozina, T. van Driel, M. Chollet, T. Sato, J. M. Glownia, S. Wandel, M. Radovic, U. Staub, and M. C. Hoffmann, Struct. Dyn. 4, 054301 (2017).

[16] W. J. Hehre, J. A. Pople, and A. J. P. Devaquet, J. Am. Chem. Soc. 98, 664 (1976).

[17] D. B. Moss and C. S. Parmenter, J. Chem. Phys. 98, 6897 (1993).
[18] J. Assmann, R. V. Benten, A. Charvat, and B. Abel, J. Phys. Chem. A 107, 1904 (2003).

[19] Q. Li, A. J. Zaczek, T. M. Korter, J. A. Zeitler, and M. T. Ruggiero, Chem. Commun. 54, 5776 (2018).

[20] See Supplemental Material at http://link.aps.org/ supplemental/10.1103/PhysRevLett.125.103001 for additional details related to the experimental and theoretical methods, x-ray diffraction structural information and Cambridge Crystallographic Data Centre (CCDC) deposition numbers, vibrational mode assignment, electron density profile determination, electron density profiles for each studied temperature, and additional information regarding the TLS analysis, which includes Refs. [21-28].

[21] G. M. Sheldrick, Acta Crystallogr. Sect. A 71, 3 (2015).

[22] G. M. Sheldrick, Acta Crystallogr. C 71, 3 (2015).

[23] L. J. Farrugia, J. Appl. Crystallogr. 32, 837 (1999).

[24] Y. C. Shen, P. F. Taday, and M. Pepper, Appl. Phys. Lett. 92, 051103 (2008).

[25] H. J. Monkhorst and J. D. Pack, Phys. Rev. B 13, 5188 (1976).

[26] F. Pascale, C. M. Zicovich-Wilson, F. L. Gejo, B. Civalleri, R. Orlando, and R. Dovesi, J. Comput. Chem. 25, 888 (2004).

[27] C. M. Zicovich-Wilson, F. Pascale, C. Roetti, V. R. Saunders, R. Orlando, and R. Dovesi, J. Comput. Chem. 25, 1873 (2004).

[28] Y. Noël, C. M. Zicovich-Wilson, B. Civalleri, P. D’Arco, and R. Dovesi, Phys. Rev. B 65, 014111 (2001).

[29] V. Schomaker and K. N. Trueblood, Acta Crystallogr. Sect. B 54, 507 (1998).

[30] Y. C. Shen, P. C. Upadhya, E. H. Linfield, and A. G. Davies, Appl. Phys. Lett. 82, 2350 (2003).

[31] M. Walther, B. M. Fischer, and P. U. Jepsen, Chem. Phys. 288, 261 (2003).

[32] R. Dovesi, A. Erba, R. Orlando, C. M. Zicovich-Wilson, B. Civalleri, L. Maschio, M. Rérat, S. Casassa, J. Baima, S. Salustro, and B. Kirtman, Comput. Mol. Sci. 8, e1360 (2018).

[33] J. P. Perdew, K. Burke, and M. Ernzerhof, Phys. Rev. Lett. 77, 3865 (1996).

[34] S. Grimme, J. Anthony, S. Ehrlich, and H. Krieg, J. Chem. Phys. 132, 154104 (2010).

[35] S. Grimme, S. Ehrlich, and L. Goerigk, J. Comput. Chem. 32, 1456 (2011).

[36] F. Weigend and R. Ahlrichs, Phys. Chem. Chem. Phys. 7, 3297 (2005).

[37] M. K. Kesharwani, B. Brauer, and J. M. L. Martin, J. Phys. Chem. A 119, 1701 (2015).

[38] R. D. Johnson, K. K. Irikura, R. N. Kacker, and R. Kessel, J. Chem. Theory Comput. 6, 2822 (2010).

[39] A. Erba, J. Maul, M. Ferrabone, P. Carbonniere, M. Rérat, and R. Dovesi, J. Chem. Theory Comput. 15, 3755 (2019).

[40] A. Erba, J. Maul, M. Ferrabone, R. Dovesi, M. Rérat, and P. Carbonniere, J. Chem. Theory Comput. 15, 3766 (2019). 